\title{
The chemical evolution of barium and europium in the Milky Way
}

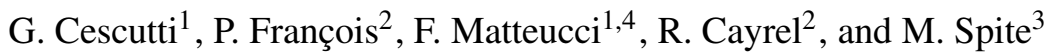 \\ 1 Dipartimento di Astronomia, Universitá di Trieste, via G.B. Tiepolo 11, 34131 Trieste, Italy \\ e-mail: cescutti@ts.astro.it \\ 2 Observatoire de Paris/Meudon, GEPI, 61 Avenue de l'Observatoire, 75014 Paris, France \\ 3 Observatoire de Paris-Meudon, GEPI, 92195 Meudon Cedex, France \\ ${ }^{4}$ I.N.A.F. Osservatorio Astronomico di Trieste, via G.B. Tiepolo 11, 34131 Trieste, Italy
}

Received 13 June 2005 / Accepted 17 October 2005

\begin{abstract}
Aims. We compute the evolution of the abundances of barium and europium in the Milky Way and we compare our results to the observed abundances from the recent UVES Large Program "First Stars".

Methods. We use a chemical evolution model that reproduces the majority of observational constraints.

Results. We confirm that barium is a neutron capture element mainly produced in the low mass AGB stars during the thermal-pulsing phase by the ${ }^{13} \mathrm{C}$ neutron source, in a slow neutron capture process. However, in order to reproduce the $[\mathrm{Ba} / \mathrm{Fe}] \mathrm{vs}$. $[\mathrm{Fe} / \mathrm{H}]$ as well as the $\mathrm{Ba}$ solar abundance, we suggest that $\mathrm{Ba}$ is also produced as an r-process element by massive stars in the range 10-30 $M_{\odot}$. On the other hand, europium should be only an r-process element produced in the same range of masses (10-30 $M_{\odot}$ ), at variance with previous suggestions indicating a smaller mass range for the Eu producers. As it is well known, there is a large spread in the $[\mathrm{Ba} / \mathrm{Fe}]$ and $[\mathrm{Eu} / \mathrm{Fe}] \mathrm{ratios}$ at low metallicities, although smaller in the newest data. With our model we estimate for both elements $(\mathrm{Ba}$ and $\mathrm{Eu})$ the ranges for the r-process yields from massive stars that better reproduce the trend of the data. We find that with the same yields able to explain the observed trends, the large spread in the $[\mathrm{Ba} / \mathrm{Fe}]$ and $[\mathrm{Eu} / \mathrm{Fe}]$ ratios cannot be explained even in the context of an inhomogeneous models for the chemical evolution of our Galaxy. We therefore derive the amount by which the yields should be modified to fully account for the observed spread. We then discuss several possibilities to explain the size of the spread. We suggest that the production ratio of [Ba/Eu] could be almost constant in massive stars.
\end{abstract}

Key words. nuclear reactions, nucleosynthesis, abundances - stars: abundances - Galaxy: abundances

\section{Introduction}

Neutron capture is the main mechanism that forms elements heavier than iron. The other mechanism, the p-process, is required for proton-rich isotopes, whose abundances in the solar system is less than $1 \%$. Two major neutron capture mechanisms are generally invoked: the slow process (s-process) and the rapid process (r-process), where the slow and rapid are defined relative to the timescale of $\beta$-decay.

The s-process requires a relatively low neutron density and moves along the valley of $\beta$ stability. The s-process feeds in particular the elements $\mathrm{Sr}-\mathrm{Y}-\mathrm{Zr}$, Ba-La-Ce-Pr-Nd and $\mathrm{Pb}$, the three major abundance s-peaks. The reason for the existence of these peaks is that: the neutron capture process imposes certain features on the "spectrum" of the heavy element abundances. For certain neutron numbers $N=50,82,126$ the neutron capture cross-sections are much smaller than for neighbouring neutron numbers. This means that once one of these "magic" numbers is reached, it becomes significantly less likely for the nucleus to capture more neutrons. These numbers are a quantum mechanical effect of closed shells, in precisely the same way that closed electron shells produce high chemical stability in the noble gases. Therefore, if the neutron capture process operates in some environment for some finite length of time and then shuts off, we expect a fair number of nuclei to be "stuck" at these "magic" numbers. Elements that correspond to these "magic" numbers of neutrons will thus be especially abundant. We then identify three peaks, as described above.

The site of production of the s-elements is not unique. The main component, accounting for the s-process in the atomic mass number range $90<A<208$, was shown to occur in the low-mass asymptotic giant branch (AGB) stars during recurrent thermal pulses (Gallino et al. 1998; Busso et al. 1999). In particular, the main s-component is due to low metallicity $([\mathrm{Fe} / \mathrm{H}]<-1.5)$ low mass AGB stars $\left(1.5-3.0 M_{\odot}\right)$.

The s-process mechanism operating in the AGB model is dependent on the initial stellar metallicity. In fact, although the ${ }^{13} \mathrm{C}$ pocket, which acts as a neutron producer, is of "primary origin" in the work of Gallino et al. (1998) and Busso et al. $(1999,2001)$, the ensuing s-process production is dependent on the initial abundance of the Fe-group seeds, i.e. on the stellar metallicity. The neutron exposure (the neutron flux per nucleus seed) is roughly proportional to the number of available 
neutron sources (the ${ }^{13} \mathrm{C}$ nuclei) per seed (the iron nuclei), hence inversely proportional to the stellar metallicity.

On the other hand, the weak s-component is responsible for the s-process nuclides up to $A \simeq 90$ and it is recognized as the result of neutron capture in advanced evolution in massive stars (see Raiteri et al. 1993). Finally, the strong-s component was introduced by Clayton \& Rassbach (1967) in order to reproduce more than $50 \%$ of solar ${ }^{208} \mathrm{~Pb}$.

The r-process takes place in extremely neutron-rich environments in which the mean time between two successive neutron captures is very short, compared with the time necessary for the $\beta$-decay. Several scenarios have been proposed for the origin of r-process elements: neutrino winds in core-collapse supernovae (Woosley et al. 1994), the collapse of ONeMg cores resulting from stars with initial masses in the range 8-10 $M_{\odot}$ (Wanajo et al. 2003) and neutron star mergers (Freiburghaus et al. 1999), even if this last scenario seems to be ruled out from recent work of Argast et al. (2004) at least as the major one responsible for r-process enrichment in our Galaxy. No clear consensus has been achieved and r-process nucleosynthesis still remains uncertain. Theoretical predictions for r-process production still do not exist, with the exception of the results of Wanajo et al. (2003) and Woosley \& Hoffmann (1992). However, the results of the model of Wanajo et al. cannot be used in galactic chemical evolution models because they do not take into account fallback (after the SN explosion some material can fall back onto central collapsing neutron star) and so the amount of neutron capture elements produced is probably too high (about 2 orders of magnitude higher than the chemical evolution predictions). Furthermore, Woosley \& Hoffmann (1992) have given prescriptions for r-processes only until ${ }^{107} \mathrm{Ru}$. In order to shed light on the nature (s- and/or rprocesses) of heavy elements such as $\mathrm{Ba}$ and $\mathrm{Eu}$ one should examine the abundances of these elements in Galactic stars of all metallicities. These abundances can give us clues to interpret their nucleosynthetic origin. In the last few years a great deal of observational work on galactic stars appeared: McWilliam et al. (1995), Ryan et al. (1996), Burris et al. (2000), Fulbright (2000), Mashonkina \& Gehren (2000, 2001), Koch \& Edvardsson (2002), Honda et al. (2004), Honda et al. (2004).

One striking aspect of the data relative to both $\mathrm{Ba}$ and $\mathrm{Eu}$ is the large spread observed in the $[\mathrm{Ba} / \mathrm{Fe}]$ and $[\mathrm{Eu} / \mathrm{Fe}]$ ratios in halo stars (e.g. Mc William et al. 1995; Ryan et al. 1996). Although this spread seems to be real, it is not found for the $[\alpha / \mathrm{Fe}]$ ratios in very metal poor stars (down to $[\mathrm{Fe} / \mathrm{H}]=-4.0$, Cayrel et al. 2004). This fact suggests that the spread, if real, is a characteristic of the nuclear capture elements and not only due to an inhomogeneous mixing in the early halo phases, as suggested by several authors (Tsujimoto et al. 1999; Ishimaru \& Wanajo 1999).

Previous studies of the evolution of s- and r-process elements are from Mathews et al. (1992), Pagel \& Tautvaisiene (1997), Travaglio et al. (1999). In the Mathews et al. (1992) paper it was suggested that the observed apparent decrease of the abundance of $\mathrm{Eu}$ for $[\mathrm{Fe} / \mathrm{H}]<-2.5$ could be due to the fact that Eu originates mainly in low mass core-collapse $\mathrm{SNe}$ (7-8 $M_{\odot}$ ). Pagel \& Tautvaisiene (1997) suggested that to reproduce the observed behaviour of $\mathrm{Ba}$ it is necessary to assume that at early stages $\mathrm{Ba}$ is produced as an r-process element by a not well identified range of massive stars. A similar conclusion was reached by Travaglio et al. (1999) who showed that the evolution of $\mathrm{Ba}$ cannot be explained by assuming that this element is only an s-process element mainly formed in stars with initial masses $2-4 M_{\odot}$, but an r-process origin for it should be considered. In the former hypothesis a very late appearance of Ba should be expected, at variance with the observations indicating that $\mathrm{Ba}$ is already produced at $[\mathrm{Fe} / \mathrm{H}]=-4.0$. They suggested that low mass SNII (from 8 to $10 M_{\odot}$ ) could be responsible for the r-component of $\mathrm{Ba}$. An attempt to explain the observed spread in s- and r-elements can be found in Tsujimoto et al. (1999) and Ishimaru \& Wanajo (1999), who claim an inefficient mixing in the early galactic phases and attribute the spread to the fact that we observe the pollution due to single supernovae. Ishimaru \& Wanajo (1999) also concluded that the Eu should originate as an r-process element in stars with masses in the range $8-10 M_{\odot}$. This latter suggestion was confirmed by Ishimaru et al. (2004) by comparing model predictions with new data from Subaru indicating subsolar $[\mathrm{Eu} / \mathrm{Fe}]$ ratios in three very metal poor stars $([\mathrm{Fe} / \mathrm{H}]<-3.0)$.

In this paper we present the results of a chemical evolution model based on the original two-infall model of Chiappini et al. (1997) for the Milky Way in the latest version developed by Chiappini et al. (2003) and adopted in François et al. (2004).

We compare the predictions relative to the evolution of $\mathrm{Ba}$ and Eu with the newest data of François et al. (2005). A spread, although lower than in previous data, still exists for $[\mathrm{Ba} / \mathrm{Fe}]$ and $[\mathrm{Eu} / \mathrm{Fe}]$ at low metallicities. We attempt to give an explanation of this spread without invoking only the inhomogeneous halo mixing.

The paper is organized as follows: in Sect. 2 we present the observational data, in Sect. 3 the chemical evolution model and in Sect. 4 the adopted nucleosynthesis prescriptions are described. In Sect. 5 we present the results and in Sect. 6 some conclusions are drawn.

\section{Observational data}

We preferentially used the most recent available data based on high quality spectra collected with efficient spectrographs and 8-10 m class telescopes. In particular, for the extremely metal poor stars $([\mathrm{Fe} / \mathrm{H}]$ between -4 and -3$)$, we adopted the recent results from UVES Large Program "First Star" (Cayrel et al. 2004; François et al. 2005). This sample consists of 31 extremely metal-poor halo stars selected in the HK survey (Beers et al. 1992, 1999). We can deduce from the kinematics of these stars that they were born at very different places in the Galactic halo. This overcomes the possibility of a selection bias. The analysis is made in a systematic and homogeneous way, from very high quality data, giving abundance ratios of unprecedented accuracy in this metallicity range. For the abundances in the remaining range of $[\mathrm{Fe} / \mathrm{H}]$, we took published high quality data in the literature from various sources: Burris et al. (2000), Fulbright (2000), Mashonkina \& Gehren (2000, 2001), Koch \& Edvardsson (2002), Honda et al. (2004), Honda et al. (2004). 
All of these data are relative to solar abundances of Grevesse \& Sauval (1998).

\section{Chemical evolution model for the Milky Way}

We model the formation of the Galaxy assuming two main infall episodes: the first forms the halo and the thick disk, the second the thin disk. The timescale for the formation of the halo-thick disk is $\sim 1$ Gyr. The timescale for the thin disk is much longer, implying that the infalling gas forming the thin disk comes mainly from the intergalactic medium and not only from the halo (Chiappini et al. 1997). Moreover, the formation of the thin disk is assumed to be a function of the galactocentric distance, leading to an inside out scenario for the Galaxy disk build up (Matteucci \& François 1989). The main characteristic of the two-infall model is an almost independent evolution between the halo and the thin disk (see also Pagel \& Tautvaisienne 1995). A threshold in the star formation process (Kennicutt 1989, 1998; Martin \& Kennicutt 2001) is also adopted. The model well reproduces an extended set of observational constraints both for the solar neighborhood and for the whole disc. One of the most important observational constraints is represented by the various relations between the abundances of metals ( $\mathrm{C}, \mathrm{N}, \mathrm{O}, \alpha$-elements, iron peak elements) as functions of the $[\mathrm{Fe} / \mathrm{H}]$ abundance (see Chiappini et al. 2003). The equation below describes the time evolution of $G_{i}$, namely the mass fraction of the element $i$ in the gas:

$$
\begin{aligned}
& \dot{G}_{i}(t)=-\psi(r, t) X_{i}(r, t) \\
& +\int_{M_{L}}^{M_{B m}} \psi\left(t-\tau_{m}\right) Q_{m i}\left(t-\tau_{m}\right) \phi(m) \mathrm{d} m \\
& +A \int_{M_{B m}}^{M_{B M}} \phi\left(M_{B}\right) \cdot\left[\int_{\mu_{m}}^{0.5} f(\mu) \psi\left(t-\tau_{m 2}\right) Q_{m i}^{\mathrm{SNIa}}\left(t-\tau_{m 2}\right) \mathrm{d} \mu\right] \mathrm{d} M_{B} \\
& +(1-A) \int_{M_{B m}}^{M_{B M}} \psi\left(t-\tau_{m}\right) Q_{m i}\left(t-\tau_{m}\right) \phi(m) \mathrm{d} m \\
& +\int_{M_{B M}}^{M_{U}} \psi\left(t-\tau_{m}\right) Q_{m i}\left(t-\tau_{m}\right) \phi(m) \mathrm{d} m \\
& +X_{A_{i}} A(r, t),
\end{aligned}
$$

where $X_{i}(r, t)$ is the abundance by mass of the element $i$ and $Q_{m i}$ indicates the fraction of mass restored by a star of mass $m$ in the form of the element $i$, the so-called "production matrix" as originally defined by Talbot \& Arnett (1973). We indicate with $M_{L}$ the lightest mass that contributes to the chemical enrichment and it is set at $0.8 M_{\odot}$; the upper mass limit, $M_{U}$, is set at $100 M_{\odot}$.

The star formation rate (SFR) $\psi(r, t)$ is defined:

$\psi(r, t)=v\left(\frac{\Sigma(r, t)}{\Sigma\left(r_{\odot}, t\right)}\right)^{2(k-1)}\left(\frac{\Sigma\left(r, t_{\mathrm{Gal}}\right)}{\Sigma(r, t)}\right)^{k-1} G_{\mathrm{gas}}^{k}(r, t)$.

$v$ is the efficiency of the star formation process and is set to be $2 \mathrm{Gyr}^{-1}$ for the Galactic halo $(t<1 \mathrm{Gyr})$ and $1 \mathrm{Gyr}^{-1}$ for the

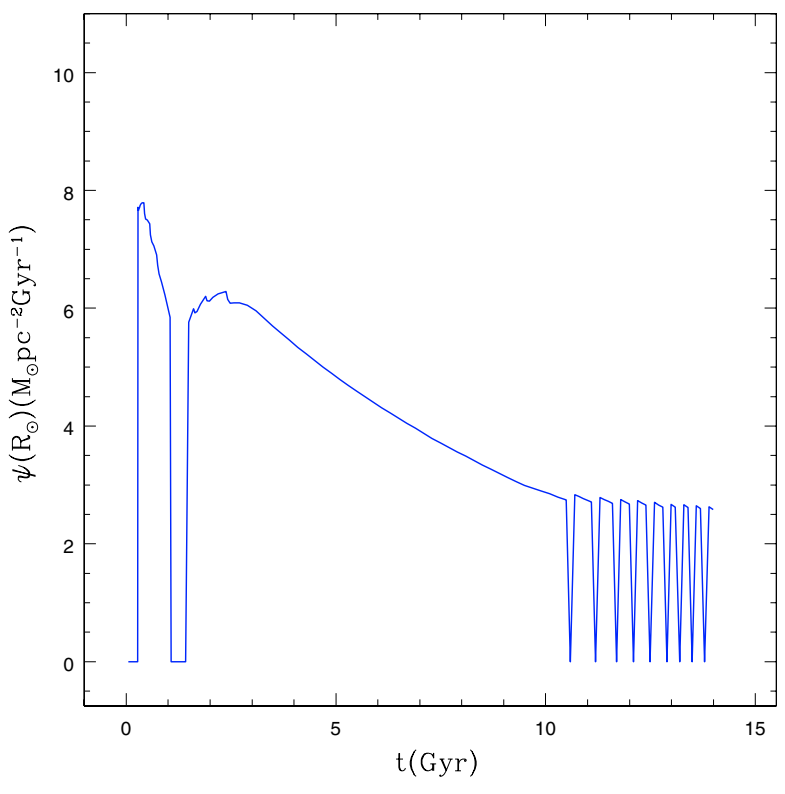

Fig. 1. The SFR expressed in $M_{\odot} \mathrm{pc}^{-2} \mathrm{Gyr}^{-1}$ as predicted by the two infall model. The gap in the SFR at the end of the halo-thick disc phase is evident. The oscillations are due to the fact that at the late times in the galactic disc the surface gas density is always close to the threshold density.

disk ( $t \geq 1$ Gyr). $\Sigma(r, t)$ is the total surface mass density, $\Sigma\left(r_{\odot}, t\right)$ the total surface mass density at the solar position, $G_{\text {gas }}(r, t)$ the surface density normalized to the present time total surface mass density in the disk $\Sigma_{D}\left(r, t_{\mathrm{Gal}}\right)$, where $t_{\mathrm{Gal}}=14 \mathrm{Gyr}$ is the age assumed for the Milky Way and $r_{\odot}=8 \mathrm{kpc}$ the solar galactocentric distance (Reid 1993). The gas surface exponent, $k$, is set equal to 1.5 . With these values for the parameters the observational constraints, in particular in the solar vicinity, are well fitted. Below a critical threshold of the gas surface density $\left(7 M_{\odot} \mathrm{pc}^{-2}\right)$ we assume no star formation. This naturally produces a hiatus in the SFR between the halo-thick disk phase and the thin disk phase. In Fig. 1 we show the predicted star formation rate for the halo-thick disc phase and the thin disc phase, respectively.

For $\phi$, the initial mass function (IMF), we use the Scalo (1986) value, constant in time and space. $\tau_{m}$ is the evolutionary lifetime of stars as a function of their mass " $m$ ".

The SNeIa rate has been computed following Greggio \& Renzini (1983) and Matteucci \& Greggio (1986) and it is expressed as:

$R_{\text {SNeIa }}=A \int_{M_{B m}}^{M_{B M}} \phi\left(M_{B}\right)\left(\int_{\mu_{m}}^{0.5} f(\mu) \psi\left(t-\tau_{M_{2}}\right) \mathrm{d} \mu\right) \mathrm{d} M_{B}$,

where $M_{2}$ is the mass of the secondary, $M_{B}$ is the total mass of the binary system, $\mu=M_{2} / M_{B}, \mu_{m}=\max \left[M(t)_{2} / M_{B},\left(M_{B}-\right.\right.$ $\left.\left.0.5 M_{B M}\right) / M_{B}\right], M_{B m}=3 M_{\odot}, M_{B M}=16 M_{\odot}$. The IMF is represented by $\phi\left(M_{B}\right)$ and refers to the total mass of the binary sistem for the computation of the SNeIa rate, $f(\mu)$ is the distribution function for the mass fraction of the secondary:

$f(\mu)=2^{1+\gamma}(1+\gamma) \mu^{\gamma}$ 


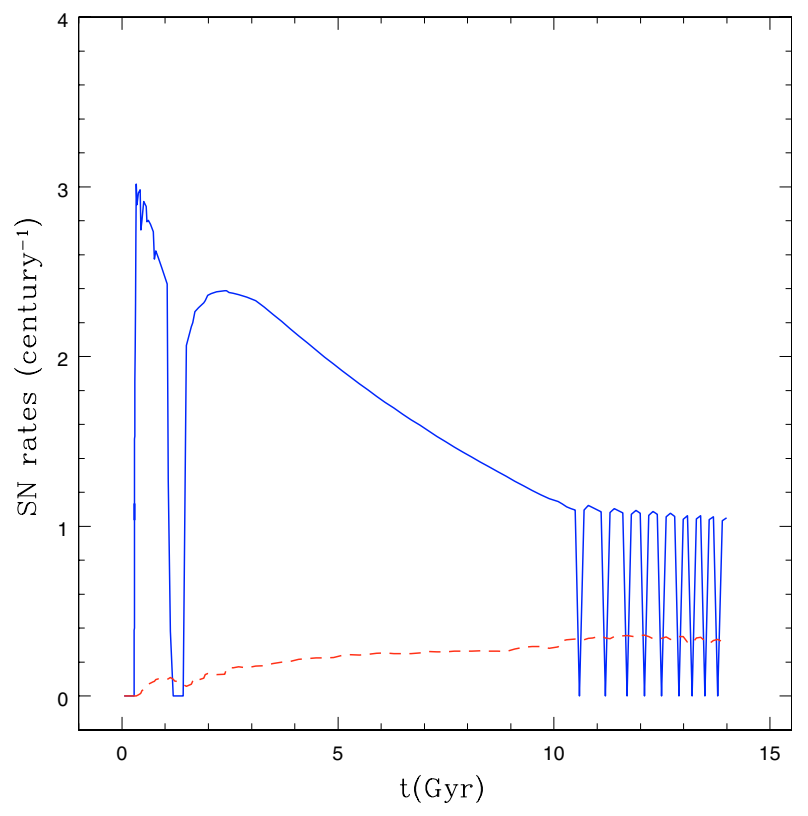

Fig. 2. Predicted SN II (continuous line) and Ia (dashed line) rates by the two infall model.

with $\gamma=2 ; A$ is the fraction of systems in the appropriate mass range that can give rise to SNeIa events. This quantity is fixed to 0.05 by reproducing the observed SNeIa rate at the present time (Cappellaro et al. 1999). Note that in the case of SNIa the "production matrix" is indicated by $Q_{m i}^{\mathrm{SNIa}}$ because of its different nucleosynthesis contribution (for details see Matteucci \& Greggio 1986). In Fig. 2 we show the predicted type II and Ia $\mathrm{SN}$ rates. The type II SN rate follows the SFR, as expected, whereas the type Ia $\mathrm{SN}$ rate does not have this feature due to the nature of type Ia SN progenitors, which are assumed to be low-intermediate mass stars with long evolutionary time scales.

The last term in Eq. (1) represents the accretion and it is defined as:

$A(r, t)=a(r) \mathrm{e}^{-t / \tau_{\mathrm{H}}}+b(r) \mathrm{e}^{\left(t-t_{\max }\right) / \tau_{\mathrm{D}}(r)}$.

$X_{A_{i}}$ are the abundances of infalling material, assumed primordial, $t_{\max }=1 \mathrm{Gyr}$ is the time for the maximum infall rate on the thin disk, $\tau_{\mathrm{H}}=2.0 \mathrm{Gyr}$ is the time scale for the formation of the halo thick-disk and $\tau_{\mathrm{D}}$ is the timescale of the thin disk, function of the galactocentric distance:

$\tau_{\mathrm{D}}=1.033 r(\mathrm{kpc})-1.267 \mathrm{Gyr}$.

The coefficients $a(r)$ and $b(r)$ are constrained by the present day total surface mass density as a function of galactocentric distance. In particular, $b(r)$ is assumed to be different from zero only for $t>t_{\max }$, where $t_{\max }$ is the time of maximum infall on the thin disc (see Chiappini et al. 2003, for details).

\section{Nucleosynthesis prescriptions}

\subsection{S-process}

We have adopted the yields of Busso et al. (2001) in the mass range $1.5-3 M_{\odot}$ for the s-main component. In this process, the dependence on the metallicity is very important. The s-process
Table 1. The stellar yields in the range $1.5-3 M_{\odot}$ from the paper of Busso et al. (2001).

\begin{tabular}{ccc}
\hline \hline Metallicity & $X_{\mathrm{Ba}}^{\text {new }}$ for $1.5 M_{\odot}$ & $X_{\mathrm{Ba}}^{\text {new }}$ for $3 M_{\odot}$ \\
\hline $0.20 \times 10^{-3}$ & $0.69 \times 10^{-8}$ & $0.13 \times 10^{-7}$ \\
$0.10 \times 10^{-2}$ & $0.38 \times 10^{-7}$ & $0.46 \times 10^{-7}$ \\
$0.20 \times 10^{-2}$ & $0.63 \times 10^{-7}$ & $0.87 \times 10^{-7}$ \\
$0.30 \times 10^{-2}$ & $0.72 \times 10^{-7}$ & $0.11 \times 10^{-6}$ \\
$0.40 \times 10^{-2}$ & $0.73 \times 10^{-7}$ & $0.12 \times 10^{-6}$ \\
$0.50 \times 10^{-2}$ & $0.68 \times 10^{-7}$ & $0.13 \times 10^{-6}$ \\
$0.60 \times 10^{-2}$ & $0.58 \times 10^{-7}$ & $0.13 \times 10^{-6}$ \\
$0.70 \times 10^{-2}$ & $0.47 \times 10^{-7}$ & $0.12 \times 10^{-6}$ \\
$0.80 \times 10^{-2}$ & $0.39 \times 10^{-7}$ & $0.11 \times 10^{-6}$ \\
$0.90 \times 10^{-2}$ & $0.34 \times 10^{-7}$ & $0.98 \times 10^{-7}$ \\
$0.10 \times 10^{-1}$ & $0.16 \times 10^{-7}$ & $0.43 \times 10^{-7}$ \\
$0.11 \times 10^{-1}$ & $0.14 \times 10^{-7}$ & $0.39 \times 10^{-7}$ \\
$0.12 \times 10^{-1}$ & $0.13 \times 10^{-7}$ & $0.34 \times 10^{-7}$ \\
$0.13 \times 10^{-1}$ & $0.12 \times 10^{-7}$ & $0.32 \times 10^{-7}$ \\
$0.14 \times 10^{-1}$ & $0.11 \times 10^{-7}$ & $0.29 \times 10^{-7}$ \\
$0.15 \times 10^{-1}$ & $0.99 \times 10^{-8}$ & $0.27 \times 10^{-7}$ \\
$0.16 \times 10^{-1}$ & $0.90 \times 10^{-8}$ & $0.25 \times 10^{-7}$ \\
$0.17 \times 10^{-1}$ & $0.81 \times 10^{-8}$ & $0.23 \times 10^{-7}$ \\
$0.18 \times 10^{-1}$ & $0.73 \times 10^{-8}$ & $0.22 \times 10^{-7}$ \\
$0.19 \times 10^{-1}$ & $0.66 \times 10^{-8}$ & $0.20 \times 10^{-7}$ \\
$0.20 \times 10^{-1}$ & $0.59 \times 10^{-8}$ & $0.19 \times 10^{-7}$ \\
$0.30 \times 10^{-1}$ & $0.24 \times 10^{-8}$ & $0.94 \times 10^{-8}$ \\
$0.40 \times 10^{-1}$ & $0.12 \times 10^{-8}$ & $0.50 \times 10^{-8}$ \\
\hline
\end{tabular}

elements are made by accretion of neutrons on seed elements (in particular iron) already present in the star. Therefore, this Ba component behaves like a secondary element. The neutron flux is due to the reaction ${ }^{13} \mathrm{C}(\alpha, n){ }^{16} \mathrm{O}$ which can easily be activated at the low temperature of these stars (see Busso et al. 1999). The yields are shown in Table 1 and Fig. 3 as functions of the initial metallicity of the stars. The theoretical results by Busso et al. (2001) suggest negligible Europium production in the s-process and therefore we neglected this component in our work. We have added for models 1 and 2 (see Table 2) an extension to the theoretical result of Busso et al. (2001) in the mass range $1-1.5 M_{\odot}$ by simply scaling with the mass the values obtained for stars of $1.5 M_{\odot}$. We have extended the prescription in order to better fit the data with a $[\mathrm{Fe} / \mathrm{H}]$ supersolar and it does not change the results of the model at $[\mathrm{Fe} / \mathrm{H}]<0$.

\subsection{R-process}

The production of r-process elements is still a challenge for astrophysics and even for nuclear physics, due to the fact that the nuclear properties of thousands of nuclei located between the valley of $\beta$ stability and the neutron drip line, necessary to correctly compute this process, are ignored. In our models we have tested 6 different nucleosynthesis prescriptions for the r-process $\mathrm{Ba}$ and $\mathrm{Eu}$, as shown in Tables 2, 3 and 4. Some of the prescriptions refer to models by Travaglio et al. (2001) (model 3) and Ishimaru et al. (2004) (models 4, 5 and 6), whereas the others contain yields chosen rather "ad hoc". 


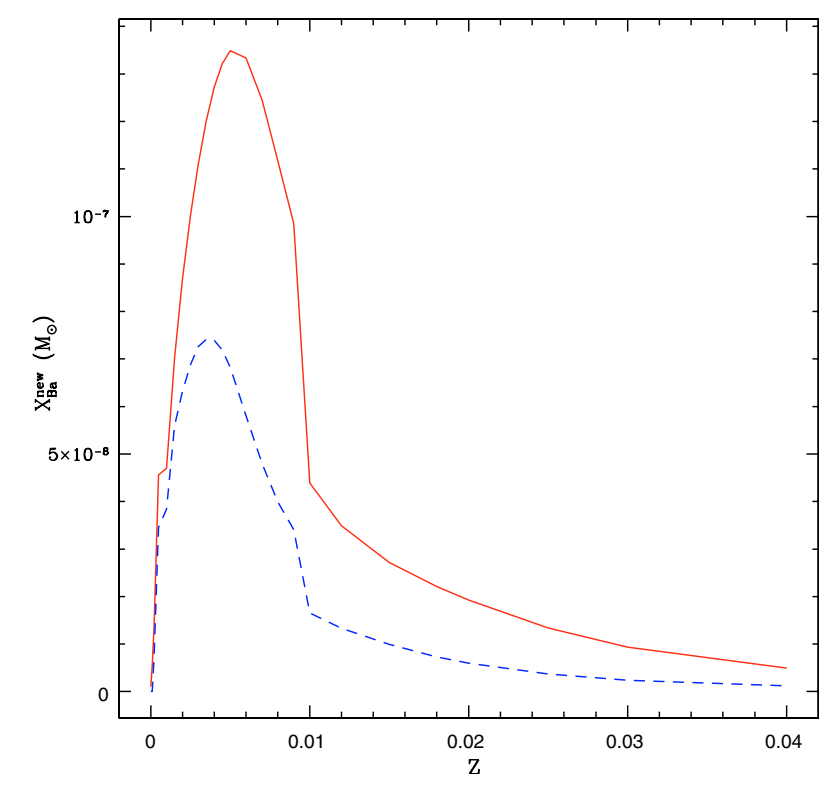

Fig. 3. The stellar yields $X_{\mathrm{Ba}}^{\text {new }}$ from Busso et al. (2001) plotted versus metallicity. Dashed line: the prescriptions for stars of $1.5 M_{\odot}$, solid line for stars of $3 M_{\odot}$.

In the case of $\mathrm{Ba}$ we have included an r-process component, produced in massive stars in the range $12-30 M_{\odot}$ in model 1 and in the range 10-25 $M_{\odot}$ in model 2. In Fig. 4 we show the lightest stellar mass dying as function of the ratio $[\mathrm{Fe} / \mathrm{H}]$ in our chemical evolution model; it is clear from this plot that it is impossible to explain the observed abundances of $[\mathrm{Ba} / \mathrm{Fe}]$ in stars with $[\mathrm{Fe} / \mathrm{H}]<-2$ without the component produced in massive stars. The first stars, which produce s-processed $\mathrm{Ba}$ (see Sect. 4.1), have a mass of $3 M_{\odot}$ and they start to enrich the ISM only at $[\mathrm{Fe} / \mathrm{H}] \geq-2$.

We stress that Travaglio et al. (2001) predicted r-process Ba only from stars in the range $8-10 M_{\odot}$, but their conclusions were based on an older set of observational data.

Moreover, we considered another independent indication for the r-process production of barium; Mazzali \& Chugai (1995) observed Ba lines in SN 1987A, which had a progenitor star of $20 M_{\odot}$. These lines of $\mathrm{Ba}$ are well reproduced with a overabundance factor $f=X_{\mathrm{obs}} / X_{i}=5$ (typical metal abundance for LMC $X_{i}=(1 / 2.75) \times$ solar $)$. From this observational data we can derive a $X_{B a}^{\text {new }} \sim 2 \times 10^{-8}$, which is in agreement with our prescriptions.

For Eu we assumed that it is completely due to the r-process and that the yields originate from massive stars in the range 12-30 $M_{\odot}$ in model 1 and $10-25 M_{\odot}$ in model 2 , as shown in Table 2.

In particular, our choice is made to best fit the plots $[\mathrm{Ba} / \mathrm{Fe}]$ vs. $[\mathrm{Fe} / \mathrm{H}],[\mathrm{Eu} / \mathrm{Fe}]$ vs. $[\mathrm{Fe} / \mathrm{H}]$ and $[\mathrm{Ba} / \mathrm{Eu}]$ vs. $[\mathrm{Fe} / \mathrm{H}]$ as well as the $\mathrm{Ba}$ and Eu solar abundance (taking into account the contribution of the low-intermediate mass star in case of the $\mathrm{Ba}$ ).

We have tested prescriptions for $\mathrm{Ba}$ and $\mathrm{Eu}$ both for a primary production and a secondary production (with a dependence on the metallicity). In the first case the main feature of the yields is a strong enhancement in the mass range $12-15 M_{\odot}$ (model 1) with no dependence on the metallicity and so the elements are considered as primary elements. In the case of metallicity dependence (model 2), the yield behaviour is chosen to have a strong enhancement in the range of metallicity $5 \times 10^{-7}<Z<1 \times 10^{-5}$ with almost constant yield for Eu and $\mathrm{Ba}$ in the whole mass range for a given metallicity.

\subsection{Iron}

For the nucleosynthesis prescriptions of $\mathrm{Fe}$, we adopted those suggested in François et al. (2004), in particular the yields of Woosley \& Weaver (1995) (hereafter WW95) for a solar chemical composition. The yields for several elements suggested by François et al. (2004) are those best reproducing the observed $[\mathrm{X} / \mathrm{Fe}]$ vs. $[\mathrm{Fe} / \mathrm{H}]$ at all metallicities in the solar vicinity.

\section{Results}

\subsection{Trends}

We investigate how the different models fit the the trends of the abundances ratios for $[\mathrm{Ba} / \mathrm{Fe}],[\mathrm{Eu} / \mathrm{Fe}]$ and $[\mathrm{Ba} / \mathrm{Eu}]$ versus $[\mathrm{Fe} / \mathrm{H}]$ and even for $[\mathrm{Ba} / \mathrm{Eu}]$ versus $[\mathrm{Ba} / \mathrm{H}]$.

To better investigate the trends of the data we divide in several bins the $[\mathrm{Fe} / \mathrm{H}]$ axis and the $[\mathrm{Ba} / \mathrm{H}]$ axis and compute the mean and the standard deviation from the mean of the ratios $[\mathrm{Ba} / \mathrm{Fe}],[\mathrm{Eu} / \mathrm{Fe}]$ and $[\mathrm{Ba} / \mathrm{Eu}]$ for all the data inside each bin.

In Table 5 we show the results of this computation for $[\mathrm{Ba} / \mathrm{Fe}]$ versus $[\mathrm{Fe} / \mathrm{H}]$, in Table 6 for $[\mathrm{Eu} / \mathrm{Fe}]$ and $[\mathrm{Ba} / \mathrm{Eu}]$ versus $[\mathrm{Fe} / \mathrm{H}]$ and in Table 7 for $[\mathrm{Ba} / \mathrm{Eu}]$ versus $[\mathrm{Ba} / \mathrm{H}]$.

Obviously having the ranges $[\mathrm{Ba} / \mathrm{H}]$ and $[\mathrm{Fe} / \mathrm{H}]$ different, we have bins of different width.

We have divided in a different way the $[\mathrm{Fe} / \mathrm{H}]$ for $[\mathrm{Ba} / \mathrm{Fe}]$ ratio and the $[\mathrm{Fe} / \mathrm{H}]$ for $[\mathrm{Eu} / \mathrm{Fe}]$ and $[\mathrm{Ba} / \mathrm{Eu}]$ ratios because the $[\mathrm{Eu} / \mathrm{Fe}]$ ratio for 12 stars at very low metallicity is only an upper limit and therefore the data for these stars have not been considered in the computation of the mean and the standard deviation for $[\mathrm{Eu} / \mathrm{Fe}]$ and $[\mathrm{Ba} / \mathrm{Eu}]$ ratios.

In the case of $[\mathrm{Ba} / \mathrm{Eu}]$ and $[\mathrm{Eu} / \mathrm{Fe}]$ we have simply divided the $[\mathrm{Fe} / \mathrm{H}]$ axis in 15 bins of equal dimension (see Table 6); for $[\mathrm{Ba} / \mathrm{Fe}]$ we have divided the $[\mathrm{Fe} / \mathrm{H}]$ in 18 bins but we have merged the first three bins (starting from the lowest value in $[\mathrm{Fe} / \mathrm{H}]$ ) into a single bin in order to have enough data in the first bin (see Table 5). For $[\mathrm{Ba} / \mathrm{Eu}]$ versus $[\mathrm{Ba} / \mathrm{H}]$ we have split the data into 16 equal bins but again we have merged the first two pairs in two bins for the same reason (see Table 7).

In Fig. 5 we show the results for the model 3 (with the yields used in Travaglio et al. 2001) for [Ba/Fe] versus [Fe/H]. As evident from Fig. 5, this model does not fit the data.

Moreover, the model in Fig. 5 is different from the similar model computed by Travaglio et al. (1999). We are using a different chemical evolution model and this gives rise to different results. The main difference between the two chemical evolution models (the one of Travaglio and the present one) is the age- $[\mathrm{Fe} / \mathrm{H}]$ relation which grows more slowly in the model of Travaglio. The cause for this difference is probably the different adopted stellar lifetimes, the different $M_{\text {up }}$ (i.e. the mass of the most massive star ending its life as $\mathrm{C}-\mathrm{O}$ white dwarf) and to the yield prescriptions for iron which are probably the 
Table 2. Model parameters. The yields $X_{\mathrm{Ba}}^{\text {new }}$ are expressed as mass fractions. The subscript "ext" stands for extended (the yields have been extrapolated down to $1 M_{\odot}$ ) and $M_{*}$ for the mass of the star.

\begin{tabular}{|c|c|c|c|c|}
\hline Mod & s-process $\mathrm{Ba}$ & r-process Ba & s-process Eu & r-process Eu \\
\hline 1 & $\begin{array}{c}1 .-3 M_{\odot} \\
\text { Busso et al. (2001) ext. }\end{array}$ & $\begin{array}{c}12-30 M_{\odot} \\
\text { yields Table } 3\end{array}$ & none & $\begin{array}{c}12-30 M_{\odot} \\
\text { yields Table } 3\end{array}$ \\
\hline 2 & $\begin{array}{c}1 .-3 M_{\odot} \\
\text { Busso et al. (2001) ext. }\end{array}$ & $\begin{array}{c}10-25 M_{\odot} \\
\text { yields Table } 4\end{array}$ & none & $\begin{array}{c}10-25 M_{\odot} \\
\text { yields Table } 4\end{array}$ \\
\hline 3 & $\begin{array}{c}1.5-3 M_{\odot} \\
\text { Busso et al. }(2001)\end{array}$ & $\begin{array}{c}8-10 M_{\odot} \\
X_{\mathrm{Ba}}^{\text {new }}=5.7 \times 10^{-6} / M_{*} \\
(\text { Travaglio et al. } 2001)\end{array}$ & none & $\begin{array}{c}12-30 M_{\odot} \\
\text { yields Table } 3\end{array}$ \\
\hline 4 & $\begin{array}{c}1.5-3 M_{\odot} \\
\text { Busso et al. }(2001)\end{array}$ & $\begin{array}{c}10-30 M_{\odot} \\
\text { yields Table } 3\end{array}$ & none & $\begin{array}{c}8-10 M_{\odot} \\
X_{\mathrm{Eu}}^{\text {new }}=3.1 \times 10^{-7} / M_{*} \\
\text { (Ishimaru et al. 2004, Mod.A) }\end{array}$ \\
\hline 5 & $\begin{array}{c}1.5-3 M_{\odot} \\
\text { Busso et al. (2001) }\end{array}$ & $\begin{array}{c}10-30 M_{\odot} \\
\text { yields Table } 3\end{array}$ & none & $\begin{array}{c}20-25 M_{\odot} \\
X_{\mathrm{Eu}}^{\text {new }}=1.1 \times 10^{-6} / M_{*} \\
\text { (Ishimaru et al. 2004, Mod.B) }\end{array}$ \\
\hline 6 & $\begin{array}{c}1.5-3 M_{\odot} \\
\text { Busso et al. (2001) }\end{array}$ & $\begin{array}{c}10-30 M_{\odot} \\
\text { yields Table } 3\end{array}$ & none & $\begin{array}{c}>30 M_{\odot} \\
X_{\mathrm{Eu}}^{\text {new }}=7.8 \times 10^{-7} / M_{*} \\
\text { (Ishimaru et al. 2004, Mod.C) }\end{array}$ \\
\hline
\end{tabular}

Table 3. The stellar yields for barium and europium in massive stars (r-process) in the case of a primary origin.

\begin{tabular}{ccc}
\hline \hline$M_{\text {star }}$ & $X_{\mathrm{Ba}}^{\text {new }}$ & $X_{\mathrm{Eu}}^{\text {new }}$ \\
\hline 12. & $9.00 \times 10^{-7}$ & $4.50 \times 10^{-8}$ \\
15. & $3.00 \times 10^{-8}$ & $3.00 \times 10^{-9}$ \\
30. & $1.00 \times 10^{-9}$ & $5.00 \times 10^{-10}$ \\
\hline
\end{tabular}

Table 4. The stellar yields for Ba and Eu in massive stars (r-process) in the case of secondary origin. The mass fraction does not change as a function of the stellar mass.

\begin{tabular}{ccc}
\hline \hline$Z_{\text {star }}$ & $X_{\text {Ba }}^{\text {new }}$ & $X_{\text {Eu }}^{\text {new }}$ \\
& $10-25 M_{\odot}$ & $10-25 M_{\odot}$ \\
\hline$Z<5 \times 10^{-7}$ & $1.00 \times 10^{-8}$ & $5.00 \times 10^{-10}$ \\
$5 \times 10^{-7}<Z<1 \times 10^{-5}$ & $1.00 \times 10^{-6}$ & $5.00 \times 10^{-8}$ \\
$Z>1 \times 10^{-5}$ & $1.60 \times 10^{-7}$ & $8.00 \times 10^{-9}$ \\
\hline
\end{tabular}

WW95 metallicity-dependent ones in the model of Travaglio et al. (1999), whereas we use the WW95 yields for the solar chemical composition, which produce a faster rise of iron.

To better fit the new data we have to extend the mass range for the production of the r-processed barium toward higher mass in order to reproduce $[\mathrm{Ba} / \mathrm{Fe}]$ at lower metallicity.

In Fig. 6, where we have plotted the predictions of model 1 and model 2 for $[\mathrm{Ba} / \mathrm{Fe}]$ versus $[\mathrm{Fe} / \mathrm{H}]$, it is clear that these models better fit the trend of the data.

In these models the upper mass limit for the production of the r-processed $\mathrm{Ba}$ is $30 M_{\odot}$ in the case of model 1 , and $25 M_{\odot}$ in the case of model 2. However, the model 2 does not fit the trend of the data as well as model 1 but the prescriptions of model 2 are very simplistic. There is no dependence on mass for a given metallicity in the yields of $\mathrm{Ba}$ and $\mathrm{Eu}$. This prescription is clearly an oversimplification but shows how a model with yields only dependent on metallicity works allowing us to estimate wheter it is appropriate or not.

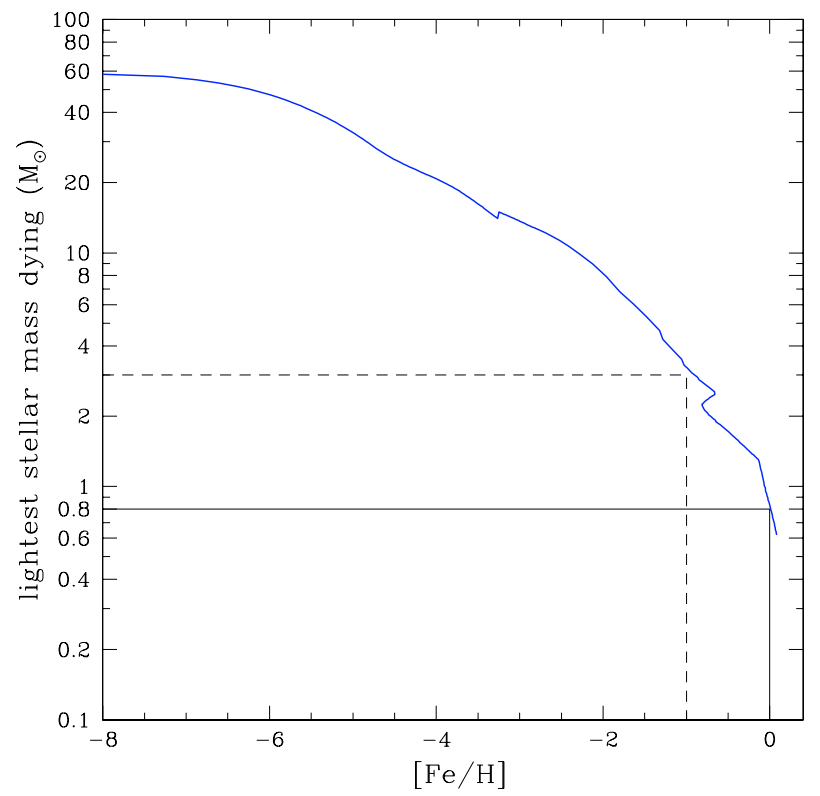

Fig. 4. In the plot we show the lightest stellar mass dying at the time corresponding to a given $[\mathrm{Fe} / \mathrm{H}]$. The solid line indicates the solar abundance $([\mathrm{Fe} / \mathrm{H}]=0)$, corresponding to a lightest dying mass star of $0.8 M_{\odot}$, the dashed line indicates the $[\mathrm{Fe} / \mathrm{H}]=-1$ corresponding to a lightest dying star mass of $3 M_{\odot}$.

We have obtained similar results comparing the trend of the abundance of $[\mathrm{Eu} / \mathrm{Fe}]$ versus $[\mathrm{Fe} / \mathrm{H}]$ with the three models of Ishimaru et al. (2004) (Model 4, 5 and 6 in Table 2). The chemical evolution of this r-process element is shown in Fig. 7. Note that that they used a different chemical model. Again model 4 does not explain the low metallicity abundances and model 5 and 6 do not fit the trend of the data well.

In Fig. 8 we show the results of models 1 and 2 in this case for $[\mathrm{Eu} / \mathrm{Fe}]$ versus $[\mathrm{Fe} / \mathrm{H}]$. The trend of the data is followed well by both models from low metallicity to solar metallicity.

In Table 8 we show the predicted solar abundances of Eu and $\mathrm{Ba}$ for all our models compared to the solar abundances 
Table 5. Results after the computation of the mean for the data inside bins along the $[\mathrm{Fe} / \mathrm{H}]$ axis for the values of $[\mathrm{Ba} / \mathrm{Fe}]$.

\begin{tabular}{ccccc}
\hline \hline Bin center $[\mathrm{Fe} / \mathrm{H}]$ & Bin dim.[Fe/H] & Mean $[\mathrm{Ba} / \mathrm{Fe}]$ & SD $[\mathrm{Ba} / \mathrm{Fe}]$ & $N$ of data in the bin \\
\hline-3.82 & 0.75 & -1.25 & 0.30 & 6 \\
-3.32 & 0.25 & -0.96 & 0.50 & 7 \\
-3.07 & 0.25 & -0.65 & 0.65 & 11 \\
-2.82 & 0.25 & -0.37 & 0.60 & 17 \\
-2.57 & 0.25 & -0.15 & 0.40 & 11 \\
-2.32 & 0.25 & 0.09 & 0.58 & 13 \\
-2.07 & 0.25 & 0.23 & 0.50 & 15 \\
-1.82 & 0.25 & 0.10 & 0.20 & 20 \\
-1.58 & 0.25 & 0.08 & 0.15 & 27 \\
-1.33 & 0.25 & 0.20 & 0.22 & 16 \\
-1.08 & 0.25 & 0.07 & 0.19 & 20 \\
-0.83 & 0.25 & -0.03 & 0.08 & 30 \\
-0.58 & 0.25 & -0.04 & 0.14 & 59 \\
-0.33 & 0.25 & 0.05 & 0.20 & 46 \\
-0.08 & 0.25 & 0.03 & 0.13 & 53 \\
0.17 & 0.25 & -0.01 & 0.11 & 26 \\
\hline
\end{tabular}

Table 6. Results after the computation of the mean for the data inside bins along the $[\mathrm{Fe} / \mathrm{H}]$ axis for the values of $[\mathrm{Eu} / \mathrm{Fe}]$ and $[\mathrm{Ba} / \mathrm{Eu}]$.

\begin{tabular}{ccccccc}
\hline \hline Bin center $[\mathrm{Fe} / \mathrm{H}]$ & Bin dim. $[\mathrm{Fe} / \mathrm{H}]$ & Mean $[\mathrm{Eu} / \mathrm{Fe}]$ & SD $[\mathrm{Eu} / \mathrm{Fe}]$ & Mean $[\mathrm{Ba} / \mathrm{Eu}]$ & SD $[\mathrm{Ba} / \mathrm{Eu}]$ & $N$ of data in the bin \\
\hline-3.22 & 0.24 & -0.10 & 0.21 & -0.71 & 0.25 & 5 \\
-2.98 & 0.24 & 0.08 & 0.60 & -0.57 & 0.13 & 12 \\
-2.74 & 0.24 & 0.46 & 0.60 & -0.64 & 0.11 & 14 \\
-2.49 & 0.24 & 0.45 & 0.28 & -0.52 & 0.17 & 7 \\
-2.25 & 0.24 & 0.38 & 0.36 & -0.38 & 0.33 & 11 \\
-2.01 & 0.24 & 0.51 & 0.34 & -0.36 & 0.26 & 10 \\
-1.77 & 0.24 & 0.29 & 0.22 & -0.20 & 0.19 & 19 \\
-1.53 & 0.24 & 0.44 & 0.15 & -0.39 & 0.22 & 21 \\
-1.28 & 0.24 & 0.42 & 0.20 & -0.26 & 0.31 & 18 \\
-1.04 & 0.24 & 0.39 & 0.13 & -0.38 & 0.15 & 16 \\
-0.80 & 0.24 & 0.32 & 0.12 & -0.35 & 0.14 & 36 \\
-0.56 & 0.24 & 0.23 & 0.14 & -0.27 & 0.20 & 55 \\
-0.32 & 0.24 & 0.18 & 0.10 & -0.13 & 0.23 & 44 \\
-0.07 & 0.24 & 0.04 & 0.07 & -0.02 & 0.14 & 51 \\
0.17 & 0.24 & -0.02 & 0.07 & 0.00 & 0.12 & 26 \\
\hline
\end{tabular}

Table 7. Results after the computation of the mean for the data inside bins along the $[\mathrm{Ba} / \mathrm{H}]$ axis for the values of $[\mathrm{Ba} / \mathrm{Eu}]$.

\begin{tabular}{ccccc}
\hline \hline Bin center $[\mathrm{Ba} / \mathrm{H}]$ & Bin dim. $[\mathrm{Ba} / \mathrm{H}]$ & Mean $[\mathrm{Ba} / \mathrm{Eu}]$ & $\mathrm{SD}[\mathrm{Ba} / \mathrm{Eu}]$ & $N$ of data in the bin \\
\hline-4.35 & 0.58 & -0.75 & 0.26 & 4 \\
-3.76 & 0.58 & -0.60 & 0.14 & 12 \\
-3.32 & 0.29 & -0.55 & 0.14 & 3 \\
-3.02 & 0.29 & -0.62 & 0.13 & 4 \\
-2.73 & 0.29 & -0.58 & 0.24 & 13 \\
-2.43 & 0.29 & -0.58 & 0.21 & 4 \\
-2.14 & 0.29 & -0.44 & 0.13 & 7 \\
-1.84 & 0.29 & -0.33 & 0.28 & 20 \\
-1.54 & 0.29 & -0.33 & 0.20 & 25 \\
-1.25 & 0.29 & -0.39 & 0.19 & 21 \\
-0.95 & 0.29 & -0.31 & 0.20 & 36 \\
-0.66 & 0.29 & -0.33 & 0.18 & 64 \\
-0.36 & 0.29 & -0.13 & 0.14 & 43 \\
-0.07 & 0.29 & -0.03 & 0.09 & 68 \\
\hline
\end{tabular}




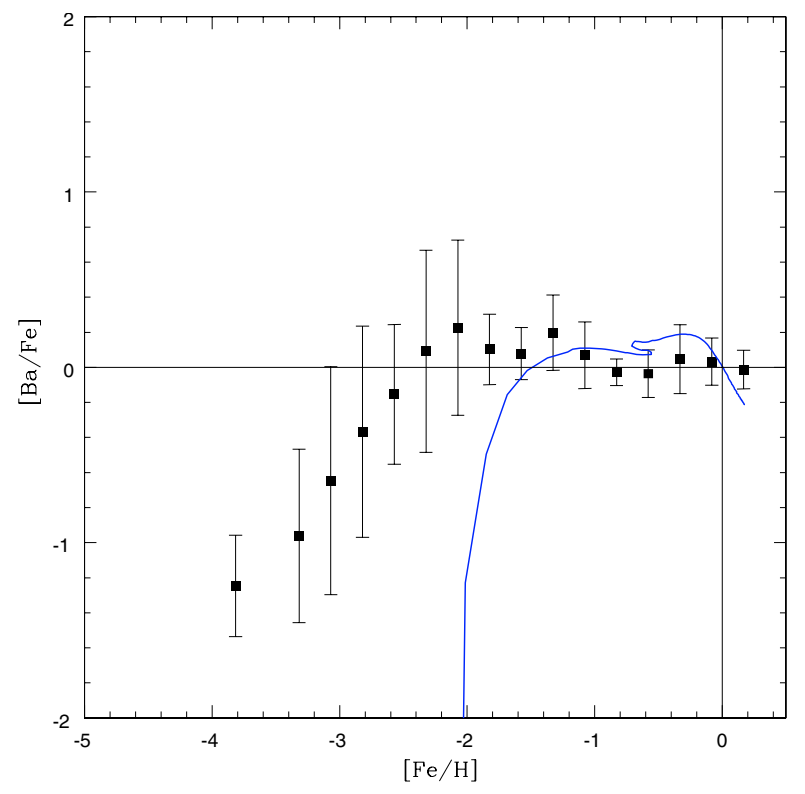

Fig. 5. The ratio $[\mathrm{Ba} / \mathrm{Fe}]$ versus $[\mathrm{Fe} / \mathrm{H}]$. The squares are the mean values of the data bins described in Table 5. For error bars we use the standard deviation (see Table 5). Solid line: the results of model 3 (Models are described in Table 2).

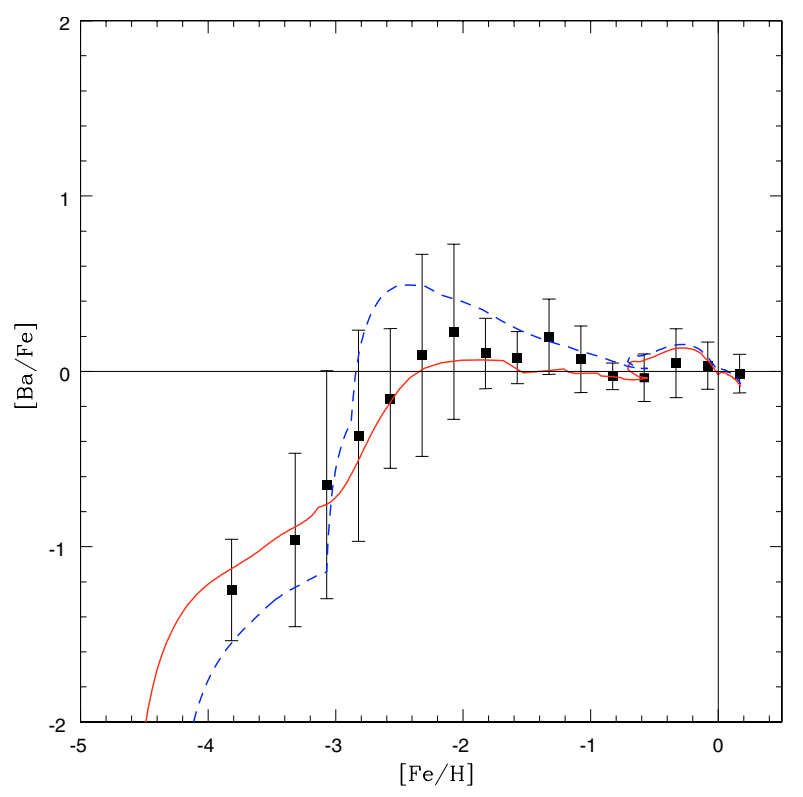

Fig. 6. The data are the same as in Fig. 5. Solid line: the model 1; dashed line: the model 2 (Models are described in Table 2).

by Grevesse \& Sauval (1998). We also give the predicted sprocess fraction in the barium solar abundance. The results of almost all our models are in good agreement with the solar abundances with the exception of model 5 which underpredicts the Eu abundance by a factor of nearly 2 . Note that we predict a slightly different s-process fraction (nearly $60 \%$ instead of $80 \%$ ) compared to the s-process fraction obtained by previous authors (Travaglio et al. 1999; Arlandini et al. 1999; Raiteri et al. 1992; Käppeler et al. 1989). This different result is again due to the adopted chemical evolution model, as discussed previously.

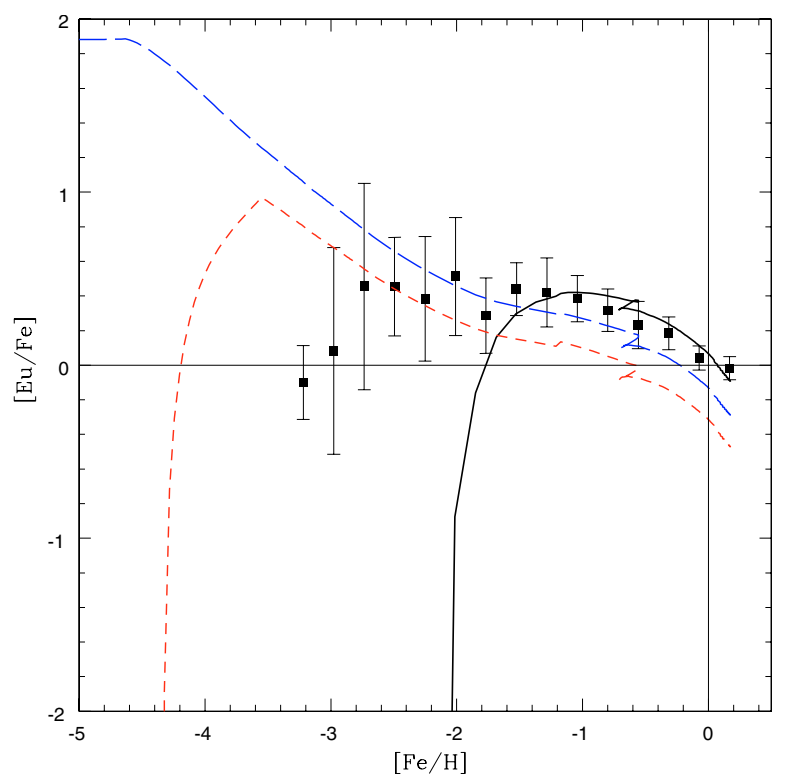

Fig. 7. $[\mathrm{Eu} / \mathrm{Fe}]$ versus $[\mathrm{Fe} / \mathrm{H}]$. The squares are the mean values of the data bins described in the Table 6. For error bars we use the standard deviation (see Table 6). Solid line: the results of model 4, short dashed line the results of model 5, long dashed line the results of model 6 (Models are described in Table 2).

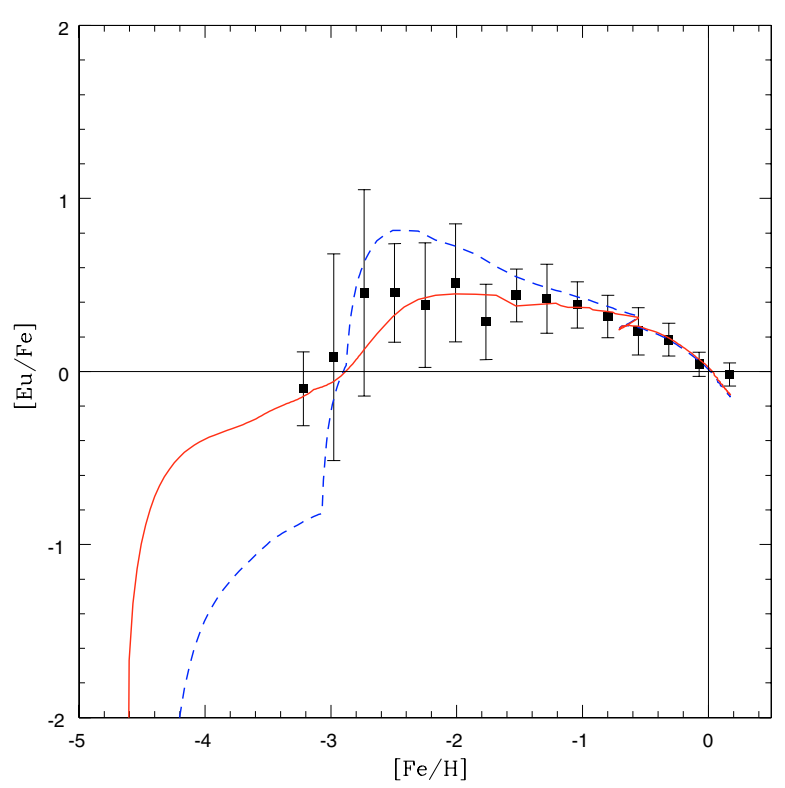

Fig. 8. Data as in Fig. 7. Solid line: the results of model 1, dashed line the results of model 2 (Models are described in Table 2).

Figure 9, where we have plotted the abundances of $[\mathrm{Ba} / \mathrm{Eu}]$ versus $[\mathrm{Fe} / \mathrm{H}]$, and Fig. 10, where we plot $[\mathrm{Ba} / \mathrm{Eu}]$ versus $[\mathrm{Ba} / \mathrm{H}]$, have three important features.

The first is that the spread, that we can infer in these plots from the standard deviation of each bin, is smaller if we use the $[\mathrm{Ba} / \mathrm{H}]$ ratio on the $x$ axis; the second feature is that it is evident from the data that there is a plateau in the $[\mathrm{Ba} / \mathrm{Eu}]$ ratio that is seen before the production of s-process $\mathrm{Ba}$ by the lowintermediate mass stars starts to be non negligible, at $[\mathrm{Fe} / \mathrm{H}] \sim$ -1 and $[\mathrm{Ba} / \mathrm{H}] \sim-0.8$; finally, the timescale of the rise of the 
Table 8. Solar abundances of $\mathrm{Ba}$ and Eu, as predicted by our models, compared to the observed ones from Grevesse \& Sauval (1998).

\begin{tabular}{cccccc}
\hline \hline Mod & $\left(X_{\mathrm{Ba}}\right)_{\mathrm{pr}}$ & $\% \mathrm{Ba}_{\mathrm{s}} / \mathrm{Ba}$ & $X_{\mathrm{Ba}_{\odot}}$ & $\left(X_{\mathrm{Eu}}\right)_{\mathrm{pr}}$ & $X_{\mathrm{Eu}_{\odot}}$ \\
\hline 1 & $1.55 \times 10^{-8}$ & $54 \%$ & $1.62 \times 10^{-8}$ & $4.06 \times 10^{-10}$ & $3.84 \times 10^{-10}$ \\
2 & $1.62 \times 10^{-8}$ & $51 \%$ & & $3.96 \times 10^{-10}$ & \\
3 & $1.64 \times 10^{-8}$ & $44 \%$ & & As model 1 & \\
4 & As model 1 & As model 1 & & $4.48 \times 10^{-10}$ & \\
5 & As model 1 & As model 1 & & $1.86 \times 10^{-10}$ & \\
6 & As model 1 & As model 1 & & $2.84 \times 10^{-10}$ & \\
\hline
\end{tabular}

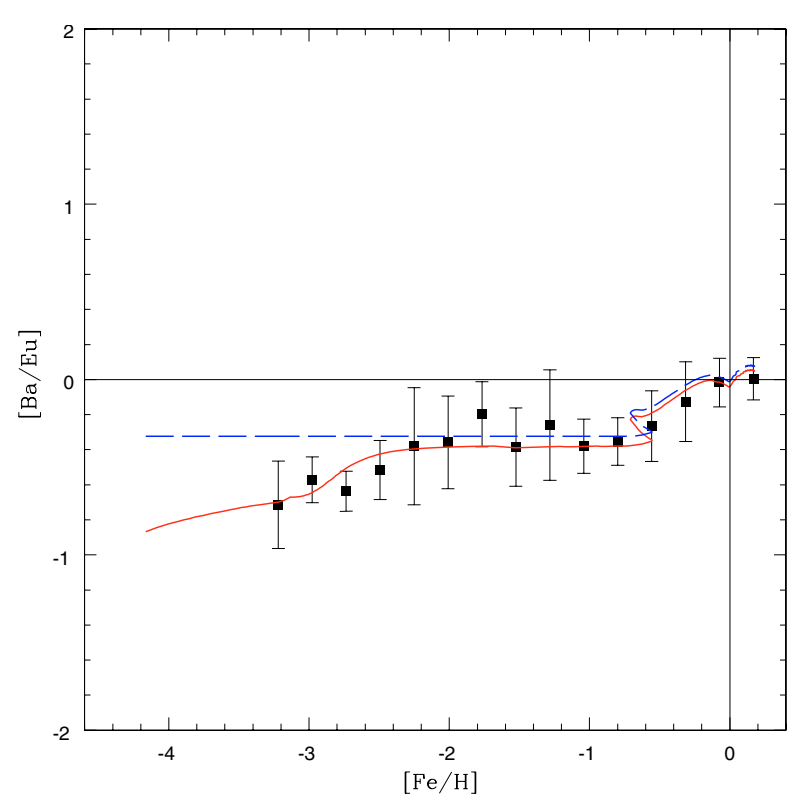

Fig. 9. The ratio of $[\mathrm{Ba} / \mathrm{Eu}]$ versus $[\mathrm{Fe} / \mathrm{H}]$. The squares are the mean values of the data bins described in Table 6. For error bars we use the standard deviation (see Table 6). Model 1: solid line, model 2: long dashed line (Models are described in Table 2).

$[\mathrm{Ba} / \mathrm{Eu}]$ value, due to the production of $\mathrm{Ba}$ by low-intermediate mass stars, is very well reproduced by our model.

The value of $[\mathrm{Ba} / \mathrm{Eu}]$ at low metallicity is important to understand the fraction of slow processed $\mathrm{Ba}$ in the solar abundance In fact, if the ratio $\frac{\mathrm{Ba}_{\text {rapid }}}{\mathrm{Eu}}$ has a constant value over cosmic time, then it must have the same value at the solar system formation time (if we do not want to add some peculiar effect during the last part of the Galaxy evolution).

In this case the Ba s-process fraction is simply:

$\frac{\mathrm{Ba}_{\text {slow }}}{\mathrm{Ba}_{\text {total }}}=1-10^{\left[\frac{\mathrm{Ba}_{\text {rapid }}}{\mathrm{Eu}}\right]}$.

Since we have a mean value for $[\mathrm{Ba} / \mathrm{Eu}]$ versus $[\mathrm{Fe} / \mathrm{H}]$ in the range $-3<[\mathrm{Fe} / \mathrm{H}]<-1$ of -0.44 (taken from the mean value in the bins which fall in that range) and a similar value for $[\mathrm{Ba} / \mathrm{Eu}]$ versus $[\mathrm{Ba} / \mathrm{H}]$ in the range $-4<[\mathrm{Ba} / \mathrm{H}]<-0.8$ of -0.41 (computed in the same way as above), then it turns out that the s-process fraction for slow processed barium has to be less than the claimed $80 \%$, with a value of $\sim 60 \%$.

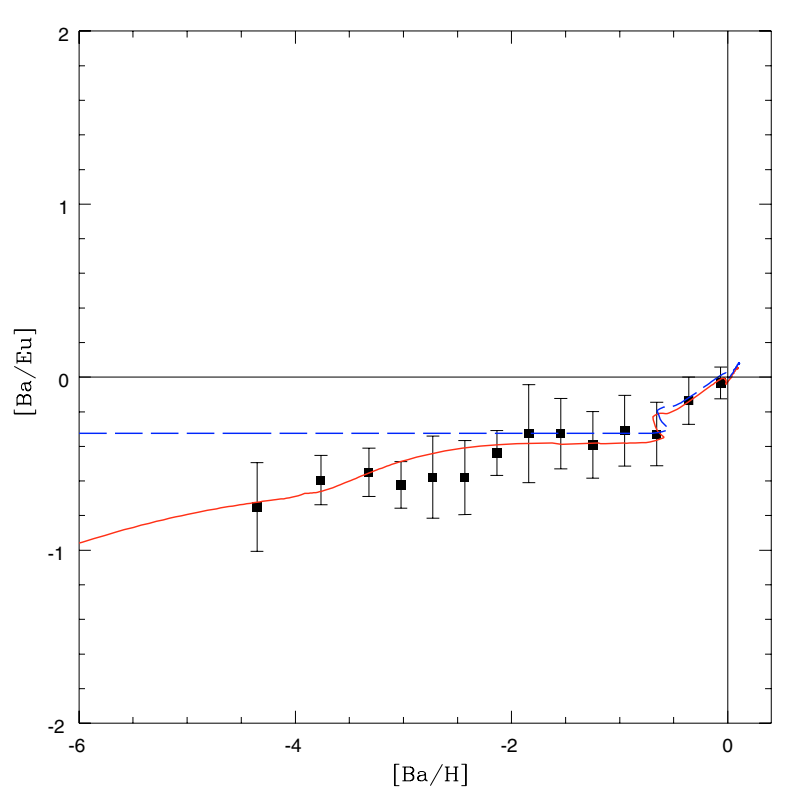

Fig. 10. $[\mathrm{Ba} / \mathrm{Eu}]$ versus $[\mathrm{Ba} / \mathrm{H}]$. The squares are the mean values of the data bins described in Table 7. For error bars we use the standard deviation (see Table 7). Model 1: solid line, model 2: long dashed line (Models are described in Table 2).

The spread in the ratio of $[\mathrm{Ba} / \mathrm{Eu}]$ both versus $[\mathrm{Fe} / \mathrm{H}]$ and $[\mathrm{Ba} / \mathrm{H}]$ is lower than the spread in $[\mathrm{Ba} / \mathrm{Fe}]$ and $[\mathrm{Eu} / \mathrm{Fe}]$, in particular when using as an evolutionary tracer the $[\mathrm{Ba} / \mathrm{H}]$.

Considering the computed standard deviations as spread tracers, where the spread for $[\mathrm{Ba} / \mathrm{Fe}]$ and $[\mathrm{Eu} / \mathrm{Fe}]$ is higher $([\mathrm{Fe} / \mathrm{H}] \sim-3)$, their standard deviations are greater than 0.6 dex whereas the standard deviations for $[\mathrm{Ba} / \mathrm{Eu}]$ is less than 0.15 dex.

For this reason we believe that the mechanism that produces the observational spread does not affect the ratio of these two elements. We propose that the explanation of the smaller spread in the ratio of $[\mathrm{Ba} / \mathrm{Eu}]$ is that the site of production of these two elements is the same: the neutronized shell close to the mass cut in a SNII (see Woosley et al. 1994). What changes could be the amount of the neutronized material that each massive star expells during the SNII explosion. The mass cut and also the ejected neutronized material are still uncertain quantities and usually they are considered as parameters in the nucleosynthesis codes for massive stars (see Rauscher et al. 2002; Woosley \& Weaver 1995; Woosley et al. 1994). 


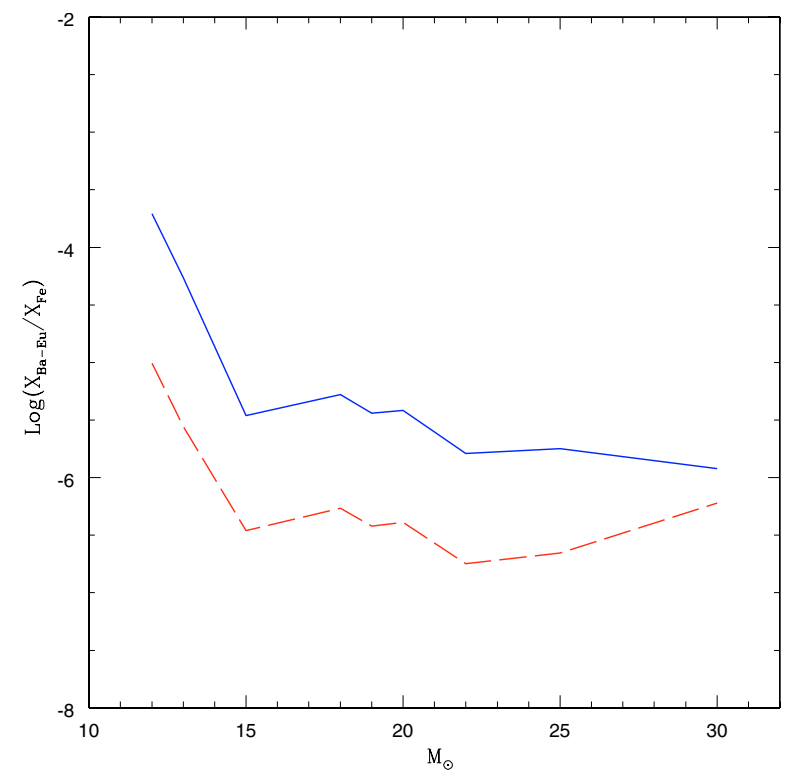

Fig. 11. The ratio between the newly produced $\mathrm{Ba}$ and $\mathrm{Fe}$ as a function of stellar mass for the model 1 yields (solid line); the same for Eu in long dashed line.

\subsection{Comparison with inhomogeneus chemical evolution models}

Our chemical evolution model is a model where instantaneous mixing is assumed, (i.e. shorter than the timestep of integration of the equations), therefore it is only able to follow the general trends of the abundance ratios as a function of $[\mathrm{Fe} / \mathrm{H}]$ found in the metal-poor stars. Inhomogeneous models assume that supernovae are able to pollute a small region of the gas surrounding them, and depending on the mass of the supernova (hence the yields) and the size of the polluted zone, an abundance spread is predicted. An important point is that the abundance ratios do not change with the amount of gas that is polluted by the supernova. It is therefore interesting to look at the range of the abundance ratios $\log (\mathrm{Ba} / \mathrm{Fe})$ that we obtain with our model and to compare them with the spread found in the observations.

In Fig. 11 we show the ratio between the r-process elements production and iron production as a function of stellar mass in the range $12-30 M_{\odot}$.

This means that the maximum spread which could be found with any inhomogeneous model of chemical evolution is shown by the range of abundance ratios displayed in Fig. 11 i.e. about 2 dex.

The observational data instead vary in a range of the order of 3 dex (see the data in Figs. 12 for $\mathrm{Ba}$ and 13 for $\mathrm{Eu}$ ). Therefore, even using an inhomogeneous model with our best model yields it would not explain the spread of the data. However, by adjusting the production of $\mathrm{Ba}$ and $\mathrm{Eu}$ only in a very narrow interval near $10 M_{\odot}$, one can account for the observed spread. This adjustment may even be justified because $\mathrm{SNe}$ of $10 M_{\odot}$ result in $\mathrm{O} / \mathrm{Mg} / \mathrm{Ne}$ core collapse and make very little $\mathrm{Fe}$, but r-process elements could be produced in the ejecta from the neutron star. A similar approach has been used in the

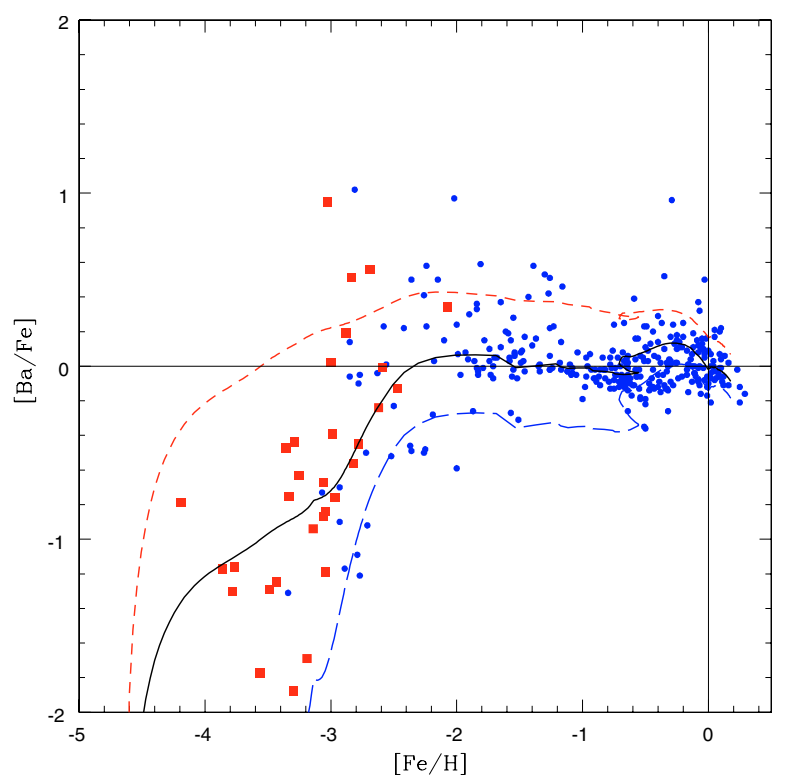

Fig. 12. $[\mathrm{Ba} / \mathrm{Fe}]$ versus $[\mathrm{Fe} / \mathrm{H}]$ for the data by François et al. (2005) (filled squares) and for the other observational data (see Sect. 2 in the text, the filled circles). The solid line is the prediction of model 1 , the short dashed line the prediction of model 1Max and the long dashed line the prediction of model $1 \mathrm{~min}$.

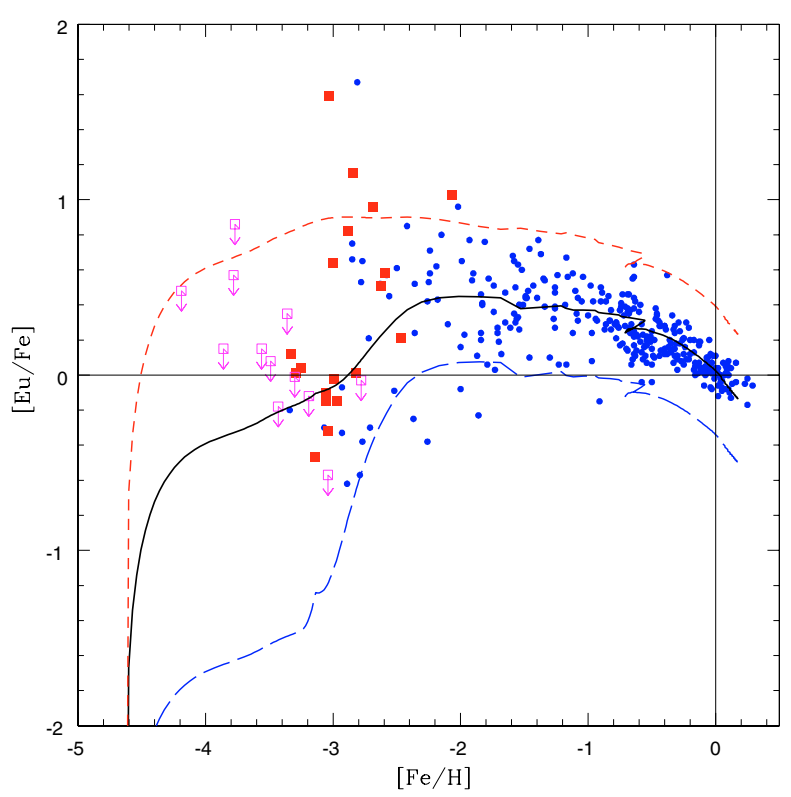

Fig. 13. $[\mathrm{Eu} / \mathrm{Fe}]$ versus $[\mathrm{Fe} / \mathrm{H}]$. The data by François et al. (2005) are filled squares, the open squares are upper limits (François et al. 2005). The filled circles are data by other observational works (see Sect. 2 in the text). The solid line is the prediction of model 1, the short dashed line the prediction of model $1 \mathrm{Max}$ and the long dashed line the prediction of model $1 \mathrm{~min}$.

inhomogeneous model of Argast et al. (2004) and by Ishimaru et al. (2004).

From the results obtained in this work, it is not possible to reproduce, even with an inhomogeneous chemical history, such a large spread in the data. It is possible that this discrepancy might be due to the existence of another parameter besides the initial mass determining the SN II yields, such as initial 
Table 9. The stellar yields for model 1Max and 1Min for barium and europium in massive stars (r-process) in the case of a primary origin.

\begin{tabular}{ccccc}
\hline \hline & Model 1Max & \multicolumn{3}{c}{ Model 1Min } \\
\hline$M_{\text {star }}$ & $X_{\mathrm{Ba}}^{\text {new }}$ & Factor & $X_{\mathrm{Ba}}^{\text {new }}$ & Factor \\
12. & $1.35 \times 10^{-6}$ & 1.5 & $4.50 \times 10^{-7}$ & 0.5 \\
$<15$. & $4.50 \times 10^{-8}$ & 1.5 & $1.50 \times 10^{-8}$ & 0.5 \\
$\geq 15$ & $3.00 \times 10^{-7}$ & 10. & $1.50 \times 10^{-9}$ & 0.05 \\
30. & $1.00 \times 10^{-8}$ & 10. & $5.00 \times 10^{-11}$ & 0.05 \\
\hline$M_{\text {star }}$ & $X_{\mathrm{Eu}}^{\text {new }}$ & Factor & $X_{\mathrm{Eu}}^{\text {nuw }}$ & Factor \\
12. & $4.50 \times 10^{-8}$ & 1. & $2.25 \times 10^{-8}$ & 0.5 \\
$<15$. & $3.00 \times 10^{-9}$ & 1. & $1.50 \times 10^{-9}$ & 0.5 \\
$\geq 15$ & $3.00 \times 10^{-8}$ & 10. & $1.50 \times 10^{-10}$ & 0.05 \\
30. & $5.00 \times 10^{-9}$ & 10. & $2.50 \times 10^{-11}$ & 0.05 \\
\hline
\end{tabular}

angular momentum. In the following section we explore what yield ratios a supernova should produce to explain both the very high and the very low [r-process/Fe] found in the sample of observed halo stars, under the assumption that the same massive stars contribute both to $\mathrm{Fe}$ and r-process synthesis, i.e. without any decoupling.

\subsection{Upper and lower limit to the r-process production}

The purpose of this section is to give upper and lower limits to the yields to reproduce the observed spread at low metallicities for $\mathrm{Ba}$ and $\mathrm{Eu}$. An inhomogeneous model would provide better predictions about the dispersion in the [r-process/Fe] ratios if due to yield variations, but it is still useful to study the effect of the yield variations by means of our model.

First we explore the range of variations of the yields as functions of the stellar mass. To do this we have worked on model 1: in particular, we have modified the yields of model 1 for both elements ( $\mathrm{Ba}$ and $\mathrm{Eu}$ ), leaving untouched the s-process yields and changing only the yields of the r-process.

$1 \mathrm{Max}$ and $1 \mathrm{~min}$ and their characteristics are summarized in Table 9.

In Figs. 12 and 13 we plot ratios $[\mathrm{Ba} / \mathrm{Fe}]$ vs. $[\mathrm{Fe} / \mathrm{H}]$ and $[\mathrm{Eu} / \mathrm{Fe}]$ vs. $[\mathrm{Fe} / \mathrm{H}]$ for the new models $1 \mathrm{Max}$ and $1 \mathrm{~min}$ compared to the observational data; we show the same plot for the ratios $[\mathrm{Ba} / \mathrm{Eu}]$ vs. $[\mathrm{Fe} / \mathrm{H}]$ in Fig. 14 and and for $[\mathrm{Ba} / \mathrm{Eu}]$ versus $[\mathrm{Ba} / \mathrm{H}]$ in Fig. 15 .

We can deduce from these upper and lower limit models that the large observed spread could also be due to a different production of heavy elements among massive stars $\left(>15 M_{\odot}\right)$. This type of star could produce different amounts of these elements independently of the mass. As we have introduced in the previous Sect. 5.1, it is possible to link this fact with the problems of mass cut and the fallback during the explosion of a SNII. If these elements are produced in a shell close to the iron core of the star, differences in the explosion behaviour can give rise to a different quantity of r-process elements expelled by the star.

In this way we are able to explain the presence of the spread for the heavy elements and the absence of the same spread for example in the $\alpha$-elements. The $\alpha$-elements are produced mostly during the hydrostatic burning of massive stars and then ejected by the explosion.

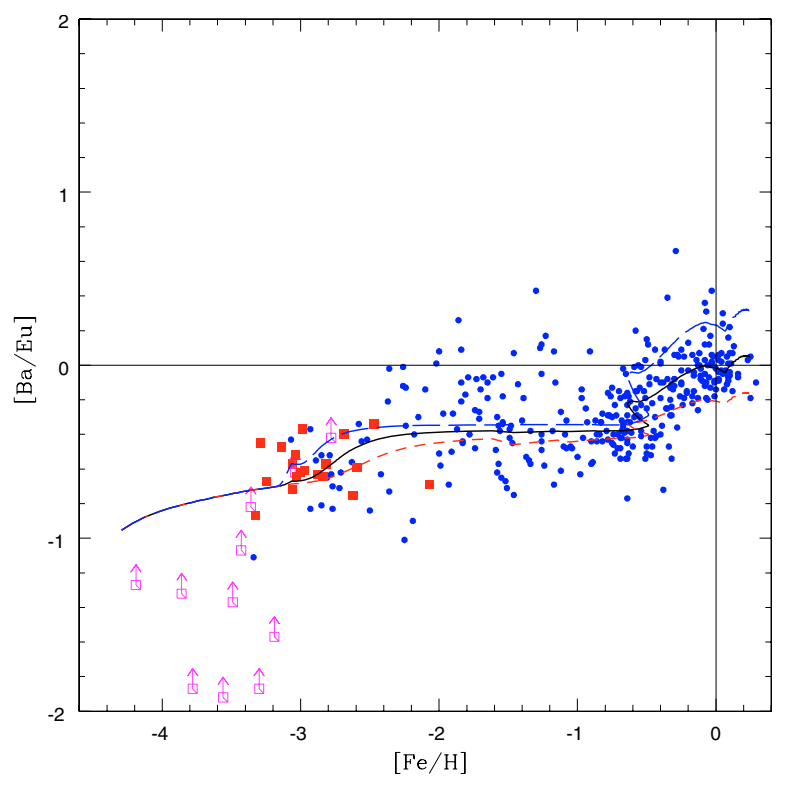

Fig. 14. $[\mathrm{Ba} / \mathrm{Eu}]$ versus $[\mathrm{Fe} / \mathrm{H}]$. The data by François et al. (2005) are filled squares, the open squares are lower limits (François et al. 2005). The filled circles are data by other observational works (see Sect. 2 in the text). The solid line is the prediction of model 1 , the short dashed line the prediction of model $1 \mathrm{Max}$ and the long dashed line the prediction of model $1 \mathrm{~min}$.

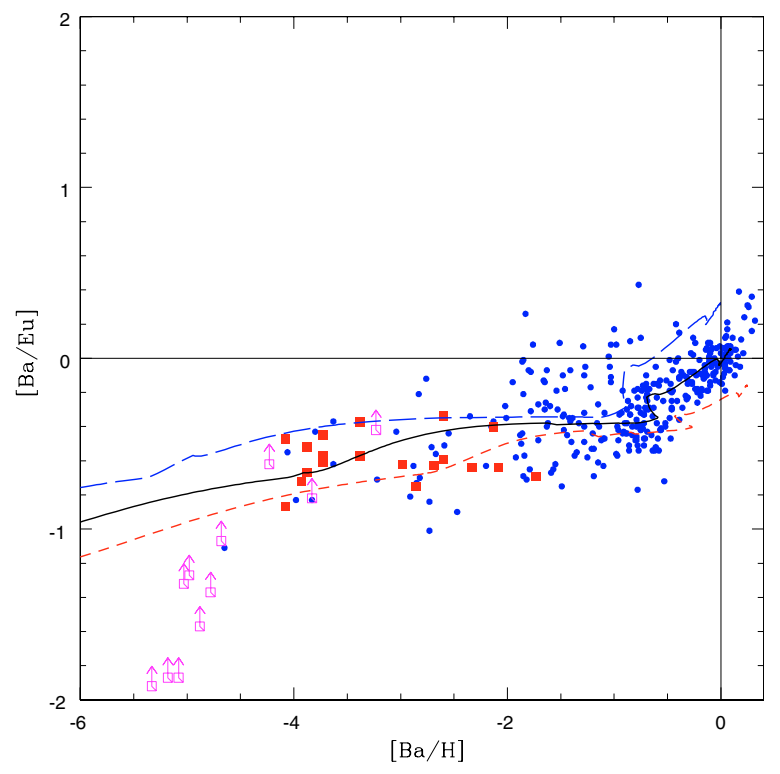

Fig. 15. As in Fig. 14 but for $[\mathrm{Ba} / \mathrm{Eu}]$ versus $[\mathrm{Ba} / \mathrm{H}]$.

Another approach can be followed to derive upper and lower limits for the model by changing the yields as functions of metallicity. Model 2, which is the model with yields independent of the mass and depending only on metallicity, will be our test model. In particular, model 2 assumes for the massive stars different yields for $\mathrm{Ba}$ and $\mathrm{Eu}$ in three ranges of metallicity (see Table 2).

The new prescriptions for both $\mathrm{Ba}$ and $\mathrm{Eu}$ are summarized in Table 10 .

In Figs. 16 and 17 we show the results of these two models (2Max and $2 \mathrm{~min}$ ) and of the original model 2 for $[\mathrm{Ba} / \mathrm{Fe}]$ 
Table 10. The stellar yields of model $2 \mathrm{Max}$ and model $2 \mathrm{~min}$ for $\mathrm{Ba}$ and $\mathrm{Eu}$ in massive stars (r-process).

\begin{tabular}{cccc}
\hline \hline$Z_{\text {star }}$ & $Z_{\text {star }}$ & $X_{\mathrm{Ba}}^{\text {new }}$ & $X_{\mathrm{Eu}}^{\text {new }}$ \\
Model 2Max & Model 2min & $10-25 M_{\odot}$ & $10-25 M_{\odot}$ \\
\hline never & $Z<8 \times 10^{-6}$. & $1.00 \times 10^{-8}$ & $5.00 \times 10^{-10}$ \\
$Z<1 \times 10^{-5}$ & $8 \times 10^{-6}<Z<1 \times 10^{-5}$ & $1.00 \times 10^{-6}$ & $5.00 \times 10^{-8}$ \\
$Z>1 \times 10^{-5}$ & $Z>1 \times 10^{-5}$ & $1.60 \times 10^{-7}$ & $8.00 \times 10^{-9}$ \\
\hline
\end{tabular}

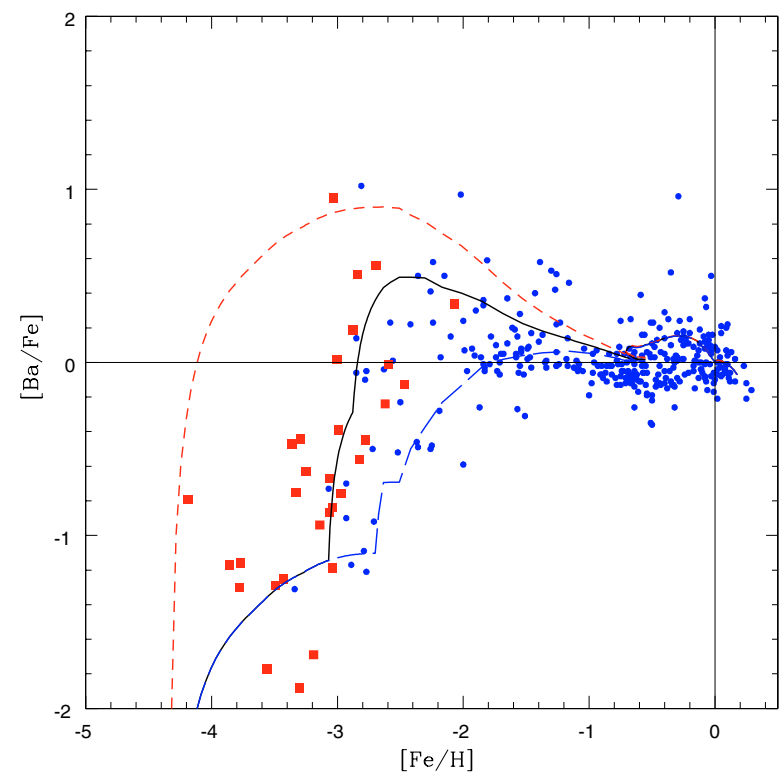

Fig. 16. $[\mathrm{Ba} / \mathrm{Fe}]$ versus $[\mathrm{Fe} / \mathrm{H}]$. The data are as in Fig. 12. The solid line is the prediction of model 2, the short dashed line the prediction of model $2 \mathrm{Max}$ and the long dashed line the prediction of model $2 \mathrm{~min}$.

vs. $[\mathrm{Fe} / \mathrm{H}]$ and for $[\mathrm{Eu} / \mathrm{Fe}]$ versus $[\mathrm{Fe} / \mathrm{H}]$ compared to the observational data.

Changing the central range of metallicity, in which there is an enhancement of the production of $\mathrm{Ba}$ and $\mathrm{Eu}$, it is possible to produce the upper and lower limits. These two new models envelope the majority of the data at low metallicities. At higher metallicity the two models overlap the best model and so they do not contain all the spread in this part of the plot but most of them could be explained inside the typical observational error of 0.1 dex.

\section{Conclusions}

The main goal of this work was to follow the evolution of $\mathrm{Ba}$ and $\mathrm{Eu}$ by means of a chemical evolution model reproducing the abundance trends for other elements. We used the Chiappini et al. (1997) model in its latest version as described in Chiappini et al. (2003). We have used empirical yields for stars with mass $>8 M_{\odot}$, producing r-process elements. For the r-process elements there are not solid theoretical yields, since the mechanism involved in their production, the so-called r-process, is still not well understood. We conclude that $\mathrm{Ba}$ needs two components: an s-process main component originating in low mass stars plus an r-component originating in stars in the range $10-30 M_{\odot}$. This range is different from the one

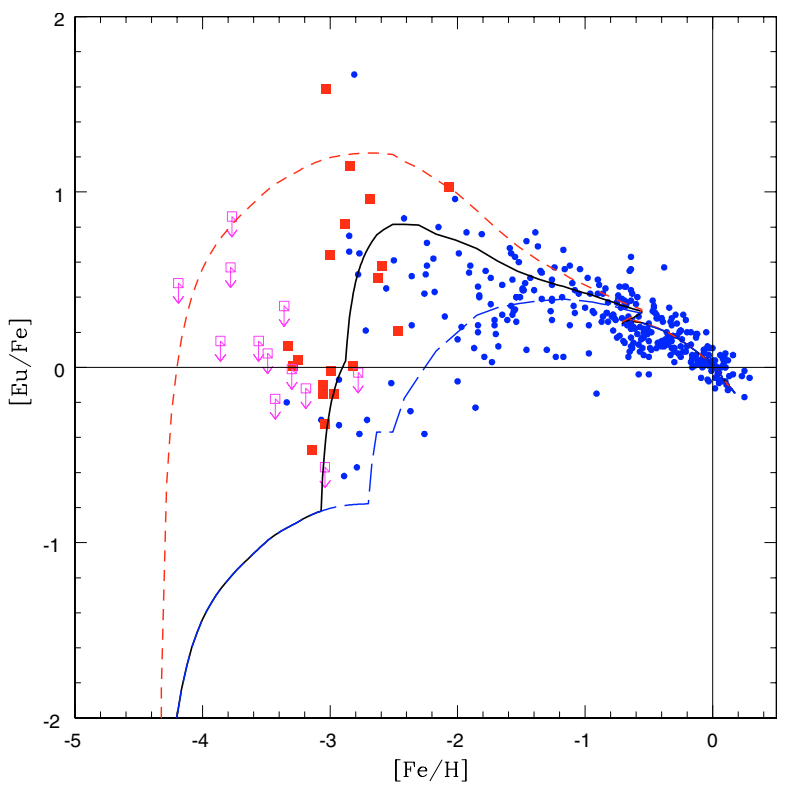

Fig. 17. $[\mathrm{Eu} / \mathrm{Fe}]$ versus $[\mathrm{Fe} / \mathrm{H}]$. The data are as in Fig. 13. The solid line is the prediction of model 2, the short dashed line the prediction of model $2 \mathrm{Max}$ and the long dashed line the prediction of model $2 \mathrm{~min}$.

suggested by Travaglio et al. (1999) and is obtained by requiring the best fit of the new data. For Eu we estimated that it is mainly produced by an r-process and that stars in the same mass range $10-30 M_{\odot}$ should be considered as the progenitors of this element.

The nearly constant value of the ratio $[\mathrm{Ba} / \mathrm{Eu}]$ produced in massive stars by the r-process can be used to estimate the fraction of barium in the solar abundance produced by the slow process. We have obtained in this way a fraction that is different from the previous results: $60 \%$ instead of $80 \%$.

We found that the yield ratios obtained to reproduce the trends are not able to explain the large spread found by the observations even with the use of an inhomogeneous model. This implies either the decoupling of the production of the r-process elements from that of $\mathrm{Fe}$ or a variation of the yields as a function of metallicity. It could be also that the yields of the rprocess are a function of the other parameters besides the mass of the progenitor.

The yields that we derived and even the fact that the ratio of the r- process production of Eu and Ba seems to be be nearly constant could be very useful in studies involving nucleosynthesis in stellar models and even in nuclear physics studies.

Acknowledgements. We thank Francesco Calura and Cristina Chiappini for several useful comments. GC and FM also acknowledge funds from MIUR, COFIN 2003, prot. N. 2003028039.

\section{References}

Argast, D., Samland, M., Thielemann, F.-K., \& Qian, Y.-Z. 2004, A\&A, 416, 997

Arlandini, C., Käppeler, F., Wisshak, K., et al. 1999, ApJ, 525, 886

Beers, T. C., Preston, G. W., \& Shectman, S. A. 1992, AJ, 103, 1987

Beers, T. C., Rossi, S., Norris, J. E., Ryan, S. G., \& Shefler, T. 1999, AJ, 117, 981 
Burris, D. L., Pilachowski, C. A., Armandroff, T. E., et al. 2000, ApJ, 544,302

Busso, M., Gallino, R., Lambert, D. L., Travaglio, C., \& Smith, V. V. 2001, ApJ, 557, 802

Busso, M., Gallino, R., \& Wasserburg, G. J. 1999, ARA\&A, 37, 239

Cappellaro, E., Evans, R., \& Turatto, M. 1999, A\&A, 351, 459

Cayrel, R., Depagne, E., Spite, M., et al. 2004, A\&A, 416, 1117

Chiappini, C., Matteucci, M. F., \& Gratton, R. G. 1997, ApJ, 477, 765

Chiappini, C., Romano, D., \& Matteucci, M. F. 2003, MNRAS, 339, 63

Clayton, D. D., \& Rassbach, M. E. 1967, ApJ, 168, 69

François, P., Depagne, E., Hill, V., et al. 2005, in preparation

François, P., Matteucci, F., Cayrel, R., et al. 2004, A\&A, 421, 613

Freiburghaus, C., Rosswog, S., \& Thielemann, F.-K. 1999, ApJ, 525, L121

Fulbright, J. P. 2000, AJ, 120, 1841

Gallino, R., Arlandini, C., Busso, M., et al. 1998, ApJ, 497, 388

Greggio, L., \& Renzini, A. 1983, A\&A, 118, 217

Grevesse, N., \& Sauval, A. J. 1998, Space Sci. Rev., 85, 161

Honda, S., Aoki, W., Kajino, T., et al. 2004, ApJ, 607, 474

Ishimaru, Y., \& Wanajo, S. 1999, ApJ, 511, L33

Ishimaru, Y., Wanajo, S., Aoki, W., \& Ryan, S. G. 2004, ApJ, 600, L47

Käppeler, F., Beer, H., \& Wisshak, K. 1989, Rep. Prog. Phys., 52, 945

Kennicutt, R. C. Jr. 1989, ApJ, 344, 685

Kennicutt, R. C. Jr. 1998, ApJ, 498, 541

Koch, A., \& Edvardsson, B. 2002, A\&A, 381, 500
Martin, C. L., \& Kennicutt, R. C. Jr. 2001, ApJ, 555, 301

Mathews, G. J., Bazan, G., \& Cowan, J. J. 1992, ApJ, 391, 719

Matteucci, M. F., \& François, P. 1989, MNRAS, 239, 885

Matteucci, M. F., \& Greggio, L. 1986, A\&A, 154, 279

Mashonkina, L., \& Gehren, T. 2000, A\&A, 364, 249

Mashonkina, L., \& Gehren, T. 2001, A\&A, 376, 232

Mazzali, P. A., \& Chugai, N. N. 1995, A\&A, 303, 118

McWilliam, A., Preston, G. W., Sneden, C., \& Searle, L. 1995, AJ, 109,2757

Pagel, B. E. J., \& Tautvaisienne, G. 1995, MNRAS, 276, 505

Pagel, B. E. J., \& Tautvaisienne, G. 1997, MNRAS, 288, 108

Raiteri, C. M., Gallino, R., \& Busso, M. 1992, ApJ, 387, 263

Raiteri, C. M., Gallino, R., \& Busso, M. 1993, ApJ, 419, 207

Rauscher, T., Heger, A., Hoffmann, R. D., \& Woosley, S. E. 2002, ApJ, 576,323

Reid, M. J. 1993, ARA\&A, 31, 345

Ryan, S., Norris, J. E., \& Beers, T. C. 1996, ApJ, 471, 254

Scalo, J. M. 1986, FCPh, 11, 1

Travaglio, C., Galli, D., Gallino, R., et al. 1999, ApJ, 521, 691

Travaglio, C., Galli, D., \& Burkert, A. 2001, ApJ, 547, 217

Tsujimoto, T., Shigeyama, T., \& Yoshii, Y. 1999, ApJ, 519, L63

Wanajo, S., Tamamura, M., Itoh, N., et al. 2003, ApJ, 593, 968

Woosley, S. E., \& Hoffmann, R. D. 1992, ApJ, 395, 202

Woosley, S. E., \& Weaver, T. A. 1995, ApJ, 101, 181

Woosley, S. E., Wilson, J. R., Mathews, G. J., Hoffman, R. D., \& Meyer, B. S. 1994, ApJ, 433, 229 